INTERNATIONAL JOURNAL OF GREEN ENERGY

Vol. 1, No. 1, pp. 47-63, 2004

\title{
Numerical Analysis of Heat Transfer and Gas Flow in PEM Fuel Cell Ducts by a Generalized Extended Darcy Model
}

\author{
Jinliang Yuan* and Bengt Sundén \\ Division of Heat Transfer, Lund Institute of Technology, Lund, Sweden
}

\begin{abstract}
In this work, gas flow and heat transfer have been numerically investigated and analyzed for both cathode/anode ducts of proton exchange membrane (PEM) fuel cells. The simulation is conducted by solving a set of conservation equations for the whole domain consisting of a porous medium, solid structure, and flow duct. A generalized extended Darcy model is employed to investigate the flow inside the porous layer. This model accounts for the boundary-layer development, shear stress, and microscopic inertial force as well. Effects of inertial coefficient, together with permeability, effective thermal conductivity, and thickness of the porous layer on gas flow and heat transfer are investigated.
\end{abstract}

Key Words: Generalized extended Darcy model; Numerical analysis; Gas flow; Heat transfer; Fuel cell.

\section{INTRODUCTION}

In recent years, the interest in all types of fuel cell for stationary, automotive, and portable power applications has increased dramatically. The understanding of fluid flow and heat transfer phenomena in fuel cells is crucial for increasing power density and most importantly, reducing manufacturing cost and accelerating

*Correspondence: Jinliang Yuan, Division of Heat Transfer, Lund Institute of Technology, Box 118, 22100 Lund, Sweden; E-mail: Jinliang.Yuan@vok.lth.se. 
commercialization of fuel cell systems. Among the various fuel cells, proton exchange membrane fuel cells (PEMFCs) have been considered as promising power sources because they can be operated at lower temperature and self-started at low temperatures, and due to low cost construction materials. Water and thermal managements are challenging issues and have to be controlled for obtaining high performance PEMFCs. Maintaining a proper water-content in the membrane is necessary to obtain acceptable ion conductivity, because less water-content will cause the membrane to be too resistive to conduct current, while more water-content can introduce cathode flooding. Consequently, a sufficient amount of water must be supplied to the anode duct to compensate for the loss due to net water transfer from the anode. On the other hand, water should be removed at a sufficient rate from the cathode duct to keep an active catalyst surface for reaction. Furthermore, the thermal management has a strong impact on the fuel cell performance, by affecting the transport of water and gaseous species as well as the electrochemical reactions in the cells.

Analyses and numerical investigations have been presented for understanding the water transport process, gas utilization, produced power, energy efficiency, electrical current, temperature distribution, and mechanical stress in both unit and stack levels of PEMFCs. Most of the models focused on one- and two-dimensional flows. Moreover, when thermal analyses and temperature distributions were included, the convective heat transfer coefficients were assumed to have constant values valid for fully developed laminar flow and available in the open literature, e.g., Lavric et al. (2001), Yi and Nguyen (1998). So far no study simulating and analyzing pressure drop and heat transfer in PEMFC ducts in terms of apparent friction factor $f_{\text {app }}$ and Nusselt number $\mathrm{Nu}$, respectively, has appeared. More comprehensive literature reviews have been reported in our works elsewhere, see Yuan (2003), Yuan et al. (2003).

Fundamental studies of forced convective heat transfer in porous media have attracted attention of various investigators in recent years (Alazmi and Vafai, 2001; Alkam et al., 2001; Bejan, 1987; Chikh et al., 1995; Comiti et al., 2000; Marafie and Vafai, 2001; Poulikakos and Renken, 1987; Teng and Zhao, 2000; Vafai and Kim, 1990, 1994). This is so because a better understanding of thermal engineering applications is required where porous materials are present. The Darcy flow model has been used for the majority of existing studies on gas flow and heat transfer in porous media, because of its simplicity and reasonably good performance within a certain range of applications. For a single-phase fully developed flow through a porous medium, the Darcy model has a linear feature, i.e., the volumetrically averaged superficial velocity in any direction in space is proportional to the net pressure gradient in that direction, and inversely proportional to the viscosity.

It is well known that the Darcy model has some limitations. It cannot predict the viscous effects and the flow development. It breaks down also due to the increasing role of inertial forces, where the interstitial flow velocity (i.e., the flow through pores of a porous medium) is not small, based on pore Reynolds number (Teng and Zhao, 2000). In the literature, there exist some studies to justify the above limitations. For example, a term accounting for friction due to macroscopic shear was included into the model to account for the transition from Darcy flow to highly viscous flow (without porous matrix, i.e., extremely high permeability). This model is 
usually referred to as the Brinkman-extended Darcy (BD) model, because the term was introduced first by Brinkman. A term representing the inertial energy of the fluid has been suggested and is often referred as the Forchheimer-extended Darcy model, see Comiti et al. (2000), Teng and Zhao (2000).

For the case of transition from porous medium flow to the pure duct flow, the permeability increases, and none of the above models takes this into account adequately. This is due to the fact that, in the high permeability limit, the terms in the momentum conservation equation can only survive for the highly viscous flows in which the effects of fluid inertia is negligible relative to pressure and friction forces (Bejan, 1987). A fully extended model to release this limitation is to combine the Forchheimer-extended Darcy model and the Navier-Stokes equations. This combined model accounts for the boundary-layer development, shear stress, and microscopic inertial force as well. As will be shown in the next section, the model is formulated for both porous medium flow and pure duct flow without the limitation of high viscosity, and it is called the generalized Brinkman-Forschheimer-Darcy model in literature, or generalized BFD model hereafter in this study. The generalized BFD model has gained large popularity partly because it enables a porous/fluid composite region to be treated as a single domain. By considering the regular fluid as a porous medium with large permeability, only one set of conservation equations needs to be solved for the entire domain. The model has been employed in fundamental studies of fluid flow and heat transfer in various porous media applications, e.g., Alazmi and Vafai (2001); Alkam et al. (2001). A more important reason for its success lies in that numerically predicted results using this formulation together with conventional boundary conditions at the interface agree well with experimental results (Vafai and Kim, 1994).

This article extends our previous investigations based on the Darcy model combined with the Navier-Stokes equations (generalized BD model hereafter) (Yuan et al., 2003), and includes the effects of the inertial force. The analysis is carried out for gas flow and heat transfer in both cathode/anode ducts by the generalized BFD model. The duct considered includes porous electrode layers, gas flow duct, and solid current collectors. Comparisons of the results from the generalized BFD model with the results of the generalized $\mathrm{BD}$ model are reported, together with other important parameter studies, such as inertial coefficient, various generated current densities, permeability, thickness, and effective thermal conductivity of the porous diffusion layers. As will be revealed later in this study, the inertial force effect has been observed for a high permeability $\left(2 \times 10^{-10} \mathrm{~m}^{2}\right)$ and/or for thick porous layer configuration $(20 \%)$ for this specific PEMFC case.

\section{MATHEMATICAL MODEL}

Figure 1 shows a schematic structure of a unit cell duct from a typical PEMFC design, which might be the anode duct for fuel gases or cathode duct for oxidant gases. The duct consists of a porous layer, gas flow duct and solid current collectors. The electrolyte is connected to the porous layers (porous electrodes) and includes thin catalyst layers on the surfaces. A steady laminar flow of an incompressible fluid is considered and all thermal-physical properties are assumed constant. In this study, 


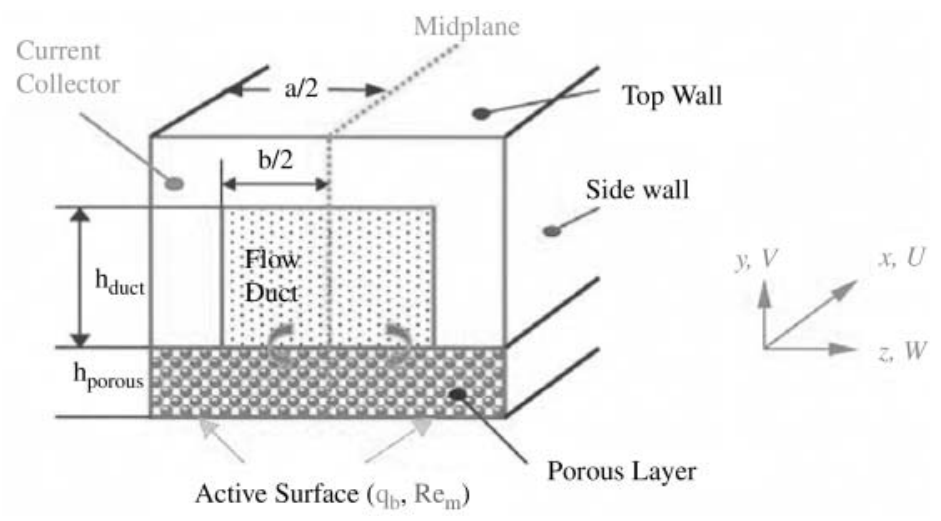

Figure 1. Schematic drawing of a fuel cell duct. (View this art in color at www.dekker.com)

the porous layers of the electrodes are assumed to be homogeneous and characterized by effective parameters, and the fluid in the porous layer is in thermal equilibrium with the solid matrix. A chemical reaction appears at the catalyst surfaces connected to the porous layer and electrolyte. The water transport at the cathode side (porous layer and cathode duct) is assumed to be in vapor form only.

The governing equations of gas flow and heat transfer are the continuity, momentum, and energy equations, which are valid for both the pure flow duct and the porous layer. These equations are written as

$$
\begin{aligned}
& \nabla \bullet\left(\rho_{\text {eff }} \mathbf{v}\right)=S_{m} \\
& \nabla \bullet\left(\rho_{\text {eff }} \mathbf{v v}\right)=-\nabla P+\nabla \bullet\left(\mu_{\text {eff }} \nabla \mathbf{v}\right)+S_{d i} \\
& \nabla \bullet\left(\rho_{\text {eff }} \mathbf{v} T\right)=\nabla \bullet\left(\frac{k_{\text {eff }}}{c_{p \text { eff }}} \nabla T\right)
\end{aligned}
$$

It should be mentioned that, for the porous layer, $\mathbf{v}$ in above equations is the volume-averaged superficial velocity vector in $x, y$, and $z$ directions. For example, the volume-averaged velocity component $U$ in the $x$ direction is equal to $\varepsilon U_{p}$, in which $\varepsilon$ is the porosity, $U_{p}$ the average pore velocity (or interstitial velocity in the literature). All thermal physical properties with subscript eff are effective ones. For the porous layer, it has been found that setting $\mu_{\mathrm{eff}}=\mu_{f}$ and $\rho_{\mathrm{eff}}=\rho_{f}$ in the equations above provide good agreement with experimental data (Poulikakos and Renken, 1987) and are adopted here as well. However, the conductivity $k_{\text {eff }}$ in the porous layer cannot be treated in a similar way (Alkam et al., 2001). Parameter studies of the effects of the effective conductivity $k_{\text {eff }}$ on the heat transfer, in terms of the conductivity ratio $k_{r}=k_{\text {eff }} / k_{f}$, have been conducted.

The source term $S_{m}$ in the mass conservation equation accounts for the electrochemical reaction effects on the mass balance and is governed by the current density. At the anode catalyst surface, the consumption of hydrogen by the electrochemical reaction and water transport due to proton transport from the anode 
side to the cathode side of the membrane (electro-osmotic drag) can be written as (Dutta et al., 2000; Shimpalee and Dutta, 2000):

$$
S_{m}=S_{\mathrm{H}_{2}}+S_{a, \mathrm{H}_{2} \mathrm{O}}=\left(-\frac{I(x, y, z)}{2 F} M_{\mathrm{H}_{2}}-\frac{\alpha(x, y, z)}{F} I(x, y, z) M_{\mathrm{H}_{2} \mathrm{O}}\right) \frac{A_{\text {active }}}{V}
$$

At the cathode catalyst surface, the source term also consists of two terms, i.e., consumption of oxygen and generation of water. It reads (Dutta et al., 2000; Shimpalee and Dutta, 2000):

$$
S_{m}=S_{\mathrm{O}_{2}}+S_{c, \mathrm{H}_{2} \mathrm{O}}=\left(-\frac{I(x, y, z)}{4 F} M_{\mathrm{O}_{2}}+\frac{1+2 \alpha(x, y, z)}{2 F} I(x, y, z) M_{\mathrm{H}_{2} \mathrm{O}}\right) \frac{A_{\text {active }}}{V}
$$

In Eqs. (4) and (5) $A_{\text {active }}$ and $V$ are the control volume surface area and volume of the active site, respectively. $\alpha(x, y, z)$ is the net water transport coefficient representing the net water transport through the membrane by electro-osmotic drag, and back diffusion due to water concentration difference, hydraulic permeation due to pressure gradient between the two sides.

The momentum equation has been formulated to be valid for both the porous layer and fluid duct, by including a source term $S_{\mathrm{di}}$.

$$
S_{\mathrm{di}}=-\left(\mu_{\mathrm{eff}} \mathbf{v} / \beta\right)-\rho_{\mathrm{eff}} B \mathbf{v}|\mathbf{v}|
$$

In the above equation, the first term on the right hand side represents for the linear relationship between the pressure gradient and flow rate by the Darcy law, while the second term is the Forchheimer term to account for the microscopic inertial effects, i.e., the nonlinear relationship between pressure drop and flow rate. The inertial coefficient $B$ in the second term is an empirical function depending on the microstructure of the porous medium, and several formulae have appeared in the literature (Bejan, 1987; Chikh et al., 1995; Marafie and Vafa, 2001; Poulikakos and Renken, 1987; Vafai and Kim, 1990). In the fluid duct, the source term $S_{\mathrm{di}}$ becomes zero because the permeability $\beta$ is infinite and $B$ is zero. Equation (2) then reduces to the regular Navier-Stokes equation. For the porous layer, the source term is not zero, and the momentum Eq. (2) with nonzero source term Eq. (6) can be regarded as a generalized Brinkman-Forchheimer-extended Darcy model, or generalized BFD model hereafter. It accounts for the macroscopic inertial effects and shear stresses, and microscopic inertial effects as well.

A constant flow rate $U=U_{\text {in }}$ is specified at the inlet for the gas flow duct, while $U=0$ is specified at the inlet for the current collector and porous layer. The boundary conditions implemented in this study can be written as:

$$
\begin{aligned}
& U=V-V_{m}=W=0, \quad-k_{\mathrm{eff}}(\partial T / \partial y)=q_{b} \quad \text { at bottom surface } \\
& U=V=W=0, \quad q=0 \quad \text { at top and side surfaces } \\
& \frac{\partial U}{\partial z}=\frac{\partial V}{\partial z}=W=\frac{\partial T}{\partial z}=0 \quad \text { at mid-plane }(z=a / 2)
\end{aligned}
$$


It is worthwhile to note that the surfaces to implement the boundary conditions have been extended to cover the solid and porous layer, see Fig. 1. In Eq. (7), $V_{m}$ is the wall velocity of mass transfer by the electrochemical reaction. The detailed procedure to obtain this value was discussed in Yuan (2003), and the final form is as follows

$$
S_{m}=\rho_{\mathrm{eff}} \operatorname{Re}_{m} \frac{v}{D_{h}} \frac{a}{A}
$$

where $\operatorname{Re}_{m}=V_{m} D_{h} / v$ is wall Reynolds number caused by the electrochemical reaction. The other variables can be found in the nomenclature list. 2000):

The heat flux $q_{b}$ is caused by the reaction and reads (Shimpalee and Dutta,

$$
q_{b}=2.45 \times 10^{5}\left[\frac{1+2 \alpha}{2 F} I(x, y, z) M_{\mathrm{H}_{2} \mathrm{O}}\right]-I(x, y, z) V_{\text {cell }}
$$

$F$ in the above equation is Faraday constant. The first term in the above equation accounts for the quantity of water, while the second term takes care of the current density generated by the electrochemical reactions. The approach used in this study is to prescribe a local current density $I(x, y, z)$ at the porous layer surface close to the catalyst layer (bottom surface in this study). Alternatively, the electrolyte including the catalyst layer should be modeled, the transport and concentration of gas species should be taken into account as well, to obtain a variable value of the local current density.

The interfacial conditions between the porous layer and flow duct have been extensively studied in the literature, e.g., Alazmi and Vafai (2001). For example, one of the early attempts revealed a slip in the velocity at the interface region by interpreting macroscopically experimental results for parallel flows. This condition is commonly called the Beavers-Joseph condition. It is worthwhile to note that this slip condition was derived from the generalized equation of motion (including both the boundary and inertial effects in the porous layer (Vafai and Kim, 1994)). As mentioned earlier, conventional boundary conditions (no-slip matching conditions) at the interface between the porous layer and flow duct, together with generalized BFD model, predict good results compared to experimental ones (Vafai and Kim, 1994). Therefore, the continuity of velocity, shear stress, temperature, and heat flux (Alazmi and Vafai, 2001) are adopted in this study:

$$
\begin{aligned}
& U_{-}=U_{+}, \quad\left(\mu_{\mathrm{eff}} \partial U / \partial y\right)_{-}=\left(\mu_{f} \partial U / \partial y\right)_{+} \\
& T_{-}=T_{+}, \quad\left(k_{\text {eff }} \partial T / \partial y\right)_{-}=\left(k_{f} \partial T / \partial y\right)_{+}
\end{aligned}
$$

in which, subscript + (plus) is for fluid side, while - (minus) is for porous layer side. Moreover, the thermal interfacial condition (Eq. (13)) is also applied at the interface between the porous medium and solid current collector with $k_{s}$ instead of $k_{f}$.

To characterize the overall pressure difference from inlet to outlet an apparent Fanning friction factor $f_{\text {app }}$ of the gas flow in a duct is defined as:

$$
f_{\text {app }}=\frac{1}{2} \frac{D_{h} d P}{\rho U_{\text {bulk }}^{2} d x}
$$


where $U_{\text {bulk }}$ is the mean velocity of the main flow, $D_{h}$ is the hydraulic diameter defined in the conventional manner, $d P / d x$ the pressure gradient along the main stream.

The bulk velocity is calculated as:

$$
U_{\text {bulk }}=\frac{\int U d A}{\int d A}
$$

and the hydraulic diameter is defined as:

$$
D_{h}=\frac{4 A}{P^{*}}
$$

$A$ is the cross-sectional area and $P^{*}$ is the wetted perimeter. It is clear that the mass transfer across the interface between the flow duct and the porous layer contributes to a change of the main flow velocity and the pressure changes accordingly along the main flow direction. Consequently, the apparent Fanning friction factor $f_{\text {app }}$ is employed in this study because it can incorporate the combined effect of wall shear and the change in momentum flow rate due to the effects of mass generation and consumption by the electrochemical reaction.

Nusselt number $\mathrm{Nu}$ at the heated wall is calculated as

$$
\overline{\mathrm{Nu}}_{b}=\frac{\bar{h}_{b} D_{h}}{k_{\mathrm{eff}}}=\frac{q_{b} D_{h}}{k_{\mathrm{eff}}\left(\bar{T}_{b}-T_{\text {bulk }}\right)}
$$

where $T_{b}$ is the bottom wall temperature; $T_{\text {bulk }}$ is the main flow mean temperature in the cross section, and calculated as

$$
T_{\text {bulk }}=\frac{\int T|U| d A}{\int|U| d A}
$$

The dimensionless axial distances $x^{*}$ and $x^{* *}$ in the flow direction for the hydrodynamic and thermal entrance regions, respectively, are defined as:

$$
\begin{aligned}
& x^{*}=x /\left(D_{h} \mathrm{Re}\right) \\
& x^{* *}=x^{*} / \operatorname{Pr}
\end{aligned}
$$

\section{NUMERICAL METHOD}

An in-house computational fluid dynamics (CFD) code is employed to solve the equations. The code is based on the finite-volume technique with boundary fitted coordinates for solving the differential equations. It should be noted that the source term in Eq. (1) accounting for mass transfer effects is zero in most of the computational domain, and nonzero only in the computational domain neighboring boundaries at the catalyst surface. New subroutines have been created to calculate the mass transfer in Eqs. (4) and (5) and the heat generation in Eq. (10). The CFD code was modified accordingly and the source term $S_{m}$ is implemented in the pressure correction equation to adjust the mass balance due to mass transfer. 
Because no flow is present in the solid current collector, Eqs. (1) and (2) are blocked out and only the heat conduction equation, reduced from the energy Eq. (3), is solved for this sub-domain.

In this investigation, a uniform control volume distribution in the cross section is applied, which was successfully adopted in previous similar studies for high temperature fuel cell ducts. To obtain finer meshes in the entrance region of the duct, a nonuniform distribution with an expansion factor is implemented in the main flow direction. Numerical calculations were carried out using various numbers of grid points and expansion factors. It has been found that the calculated apparent friction factors and Nusselt numbers do not change significantly when the number of grid points are increased beyond $81 \times 41 \times 25(x \times y \times z)$ for all cases. To validate the code, the Nusselt numbers from the present study are compared with analytical ones of the fully developed parallel channel flow, for the same boundary conditions of constant heat flux on the walls from (Poulikakos and Renken, 1987). The deviations between the simulations and analytical results are sufficiently small and it is reasonable to believe that the numerical method and code can be used reliably for the present study.

\section{CASE STUDY}

The following parameters of a PEMFC and its porous medium, appeared in the literature, and these are used as a base case in this work. The following values are valid: $V_{\text {cell }}=0.53 \mathrm{~V}, I=1.0 \times 10^{4} \mathrm{~A} / \mathrm{m}^{2}, \alpha=0.3$, gases (air for the oxidant duct, while $0.53 \mathrm{~mol}$ fraction of $\mathrm{H}_{2}$, and 0.47 of water vapor for the fuel duct) with inlet temperature $T_{\mathrm{in}}=80^{\circ} \mathrm{C} ; k_{s}=5.7 \mathrm{~W} /(\mathrm{m} \mathrm{K}), \mu_{\mathrm{eff}}=\mu_{f}, \varepsilon=0.7$, and $\beta=2.0 \times 10^{-10} \mathrm{~m}^{2}$. Similar to (Dutta et al., 2000; Shimpalee and Dutta, 2000), the dimensions of the whole channel are as follows: overall channel is $10 \mathrm{~cm} \times 0.20 \mathrm{~cm} \times 0.16 \mathrm{~cm}(x \times y \times z)$, gas flow duct is $10 \mathrm{~cm} \times 0.12 \mathrm{~cm} \times 0.08 \mathrm{~cm}(x \times y \times z)$, while the porous layer is $10 \mathrm{~cm} \times 0.04 \mathrm{~cm} \times 0.16 \mathrm{~cm}(x \times y \times z)$. For this case, the thickness ratio $h_{r}$ (thickness of porous layer $h_{\text {porous }}$ over total height $h$ of the duct) is $20 \%$. The inertial coefficient $B$ depends very much on the microstructure of the porous medium, and theoretical determination of it is not easy. In fact, the value of $B$ reported often varies between investigators and the porous medium tested. As an example, two models from the literature for $B$ are given in Table 1. It is clear that the model 2 in the table needs more detailed information about the porous medium microstructure in PEMFC, which is not available at the present moment. Only model 1 is then used in the present study. Table 2 shows methods to determine the Forchheimer coefficient $F$ in

Table 1. The inertial coefficient $B$ in Eq. (6).

\begin{tabular}{lll}
\hline Model & The inertial coefficient & \multicolumn{1}{c}{ References } \\
\hline BFD-1 & $B=\varepsilon F /(\beta)^{0.5}$ & $\begin{array}{c}\text { (Bejan, 1987; Chikh et al., 1995; Marafie and Vafai, 2001; } \\
\text { Vafai and Kim, 1994) } \\
\text { BFD-2 }\end{array}$ \\
\hline
\end{tabular}


Table 2. The Forchheimer coefficient $F$ in BFD-1 model.

\begin{tabular}{lcl}
\hline Model & The Forchheimer coefficient & \multicolumn{1}{c}{ References } \\
\hline BFD-1a & $F=1.8 /\left(180 \varepsilon^{5}\right)^{0.5}$ & (Bejan, 1987) \\
BFD-1b & $F=0.143 \varepsilon^{-1.5}$ & (Vafai and Kim, 1994) \\
\hline
\end{tabular}

model 1, and both are adopted in the study. Because the inertial effects are of main interest, a parameter study has also been conducted, see next section for details.

\section{RESULTS AND DISCUSSIONS}

In this section, the main results of the numerical simulations are reported and discussed. It should be noted that all the results presented hereafter are for the base case condition unless otherwise stated. Figure 2 a shows the velocity profiles along the main flow direction, in which the scale of the vector plots (i.e., $20 \mathrm{~m} / \mathrm{s}$ ) is a reference value of the maximum velocity. As expected, a parabolic profile is clearly observed in the flow duct. On the other hand, the velocity in the porous layer is very small except in the region close to the duct, because the gas penetration into the porous layer is weak. This finding can be clearly observed in Fig. 2b, which is an enlarged figure for the porous layer. A small convective gas flow is then identified in the porous layer of the fuel cell ducts.

Figure 3 shows cross-section velocity vectors in the porous layer and cathode flow duct for selected longitudinal stations, $1 / 3$ and $2 / 3$ of the duct length from the entrance (i.e., at $x=0.033 \mathrm{~m}$ and $x=0.067 \mathrm{~m}$ ). At the inlet region (Fig. 3a), the cross section vectors are clearly dominated by upward flow due to mass transport in the porous layer and lower part of the cathode duct. Fluid moves towards the center of the duct in the core region, which is due to the entrance effects. As mentioned above, oxygen is consumed on the catalyst surface while water vapor is produced and generated into the porous layer by the electrochemical reaction. It is known that the amount of water generated and transported is larger than the oxygen consumed. The net mass transport is similar to a mass injection to the porous layer and cathode duct, and thus there exists a secondary flow pointing upwards in the region near the catalyst surface. The position of the center moves upward until the center disappears as the flow goes further downstream (Fig. 3b), and thus the cross section velocity vectors are also upward in the cathode duct.

Figure $4 \mathrm{a}$ demonstrates the axial velocities at various positions from the entrance. It is shown that a constant $U_{\text {in }}$ is prescribed at the inlet of the duct. The fully developed velocity profile is maintained after $1 / 3$ of the duct. Comparisons of axial velocity profiles, simulated by the generalized BD and BFD models, are presented in Fig. 4b. It is clear that the velocity profiles by the generalized BFD model in the porous layer are more uniform, which was confirmed also in Chikh et al. (1995). This is due to the fact that the inertial force in Eq. (6) has a similar contribution as the Darcy force, and resembles an extra retarding force for the gas flow in the porous layer. It is noticed that the gradients of the axial velocity in the 
(a)

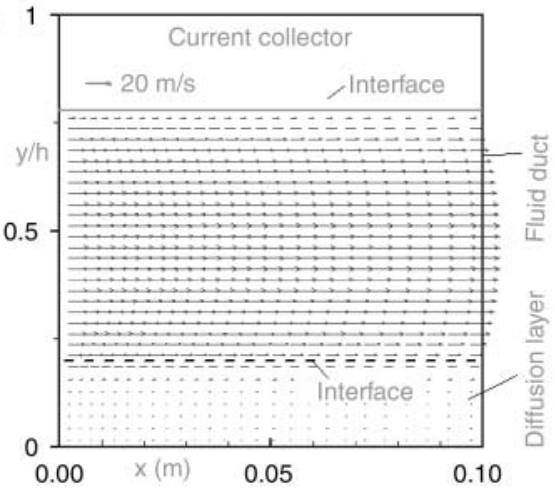

(b)

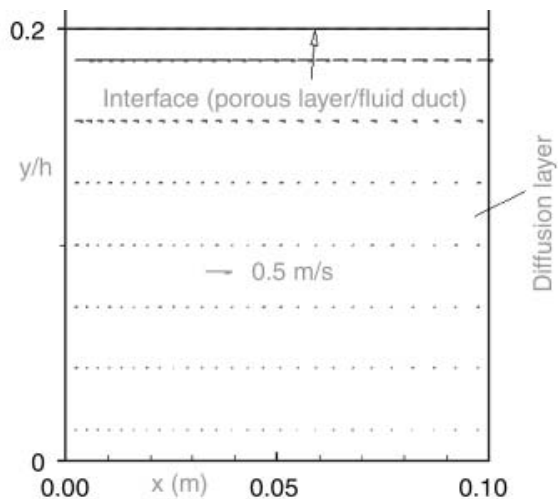

Figure 2. Velocity vectors in: (a) whole domain, and (b) porous layer of the cathode duct along the main flow predicted by the BFD-1a model. (View this art in color at www.dekker.com)

Cross-section velocity vectors

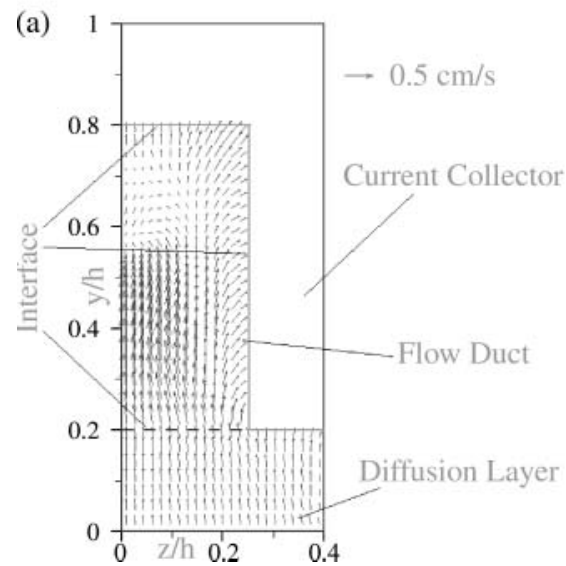

Cross-section temperature contours

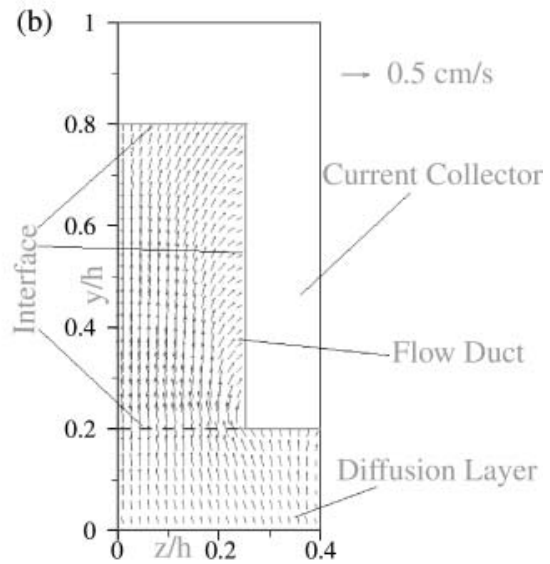

Figure 3. Cross-section velocity vectors in the porous layer and cathode gas flow duct at: (a) $1 / 3$, (b) $2 / 3$ of the duct length from the inlet. (View this art in color at www.dekker.com)

flow duct and the porous layer within the interface region are the same. This is so because the interfacial condition is based on the continuity of shear stress, and because of the assumption $\mu_{\mathrm{eff}}=\mu_{f}$ no change in the velocity gradient appears.

Figure 5 shows the variations of cross section averaged apparent friction factor $f_{\text {app }} \operatorname{Re}$ (presented as $f_{\text {app }} \operatorname{Re} / f_{d} \operatorname{Re}$, where $f_{d}$ is the one for fully developed flow) and Nusselt number $\mathrm{Nu}$ along the axial direction with various models. It is clear that both $f_{\text {app }}$ Re and Nu rapidly decay due to the hydro- and thermodynamic boundary layer development. Compared to the BD model, the generalized BFD models predict a larger $f_{\text {app }} \mathrm{Re}$ and a smaller $\mathrm{Nu}$ for the PEMFC cathode duct, see Figs. 5a and b. This is because the gas flow, retarded in the porous layer by the inertial forces, 

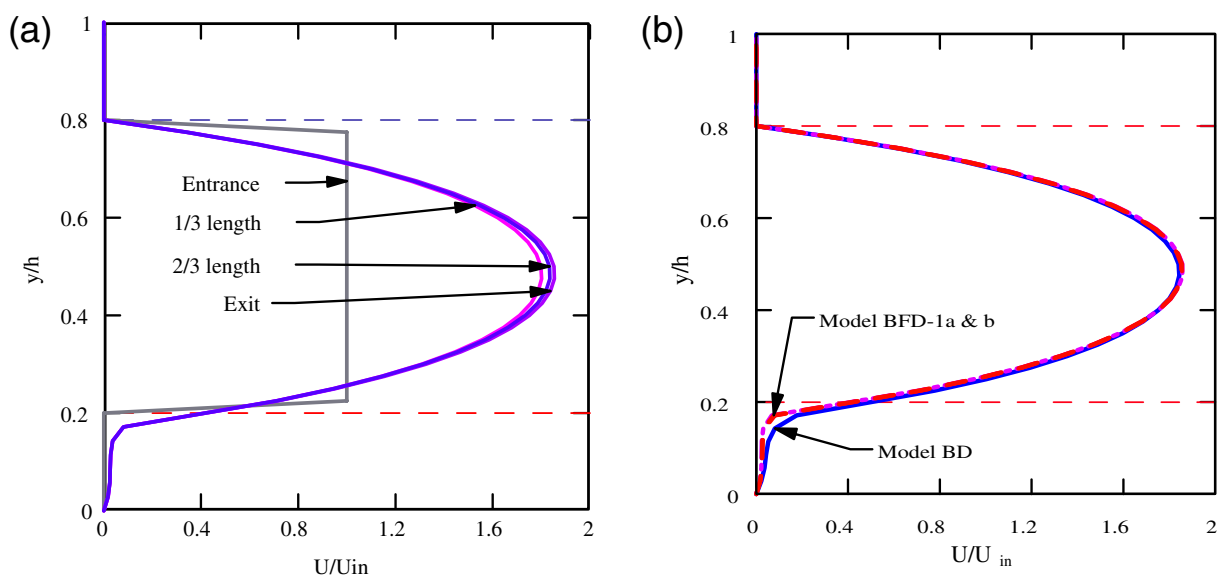

Figure 4. (a) Development and (b) profiles of axial velocity in the cathode duct predicted by various models. (View this art in color at www.dekker.com)
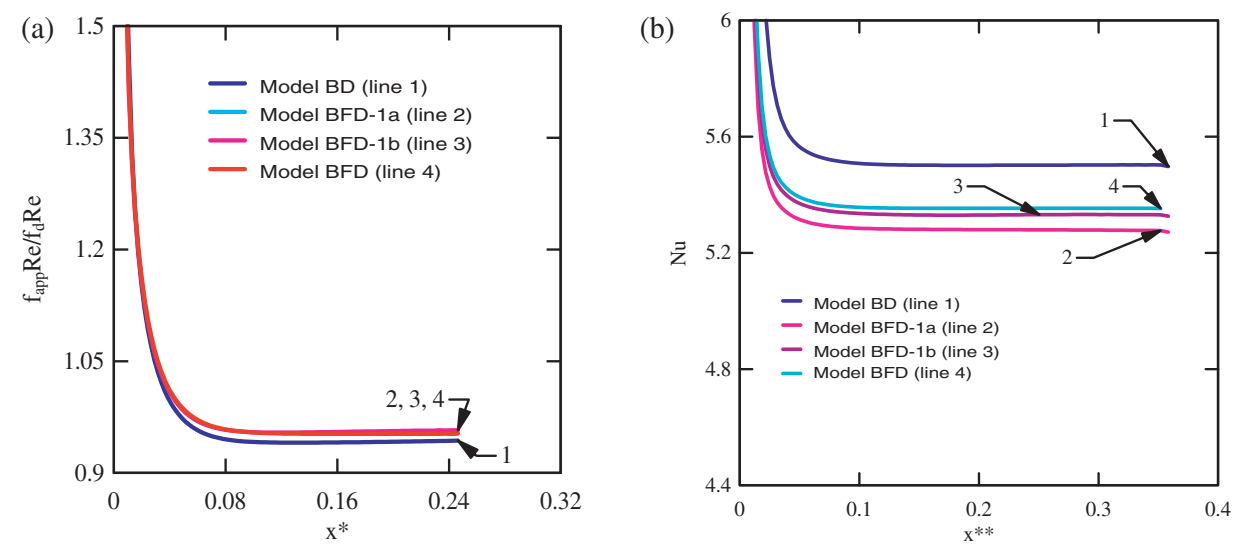

Figure 5. (a) $f_{\text {app }} \mathrm{Re}$ and (b) $\mathrm{Nu}$ of cathode duct predicted by generalized $\mathrm{BD}$ and $\mathrm{BFD}$ models. (View this art in color at www.dekker.com)

contributes with an extra velocity increase. Thus, the axial velocity and its gradient increase, similar to a mass injection into the duct. On the other hand, the retarded flow from the porous layer induces more heat into the duct. As revealed in (Yuan, 2003) the heat transfer is a combination of pure convection and energy transport by the injected flow. As a consequence the convective $\mathrm{Nu}$ is decreased due to the large injected heat. It is worthwhile to note that the type of BFD model has significant effects on the decrease of $\mathrm{Nu}$, while small effects on the increase of $f_{\text {app }} \mathrm{Re}$. It is also clear that both BFD-1a and b models produce similar results for $\mathrm{Nu}$, which are close to that at $F=10^{4}$ in the figure. From Table 2, it can be verified that the Forchheimer coefficient $F$ from both models is in the order of $10^{4}$ for this specific PEMFC case $(\varepsilon=0.7)$. 

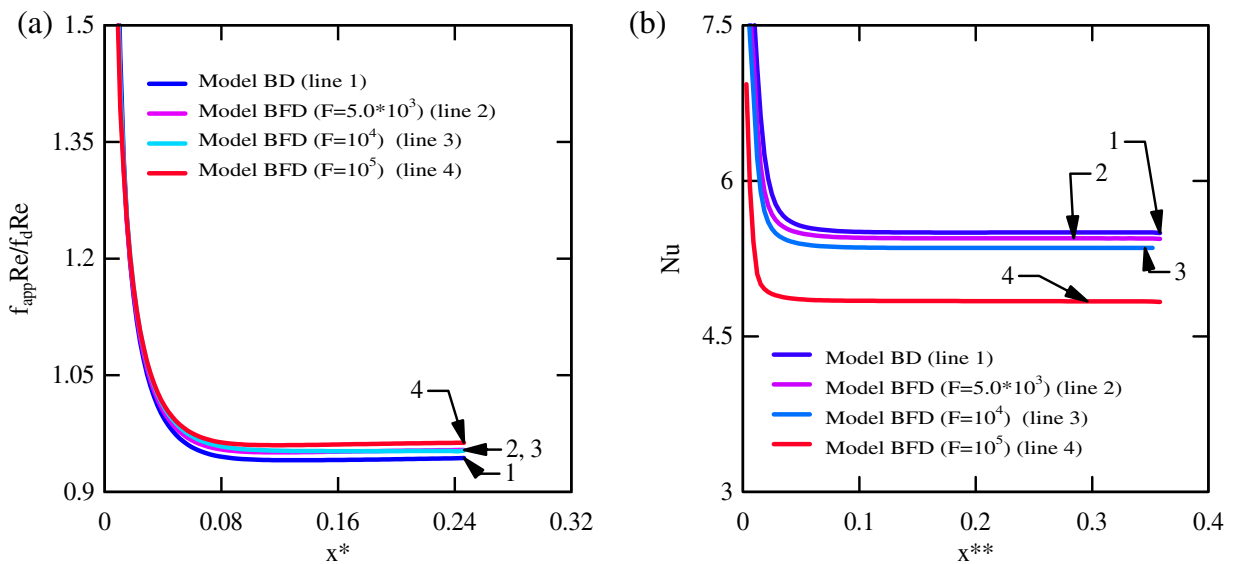

Figure 6. Effects of the inertial energy on: (a) $f_{\text {app }} \mathrm{Re}$ and (b) $\mathrm{Nu}$ of cathode duct predicted by the generalized BFD models. (View this art in color at www.dekker.com)

Parameter studies of the inertial effects, in terms of the Forchheimer coefficient $F$, have been conducted for a wide range, covering PEMFC cases. Results are shown in Figs. 6a and b. With reference to $f_{\text {app }}$ Re in Fig. 6a, it is noticed that an increase of $F$ (BFD Models vs. BD Model) can increase $f_{\text {app }}$ Re, but all the cases produce similar $f_{\text {app }}$ Re, i.e., the Forchheimer coefficient $F$ has a limited effect on $f_{\text {app }}$ Re. On the other hand, for heat transfer, significant changes of $\mathrm{Nu}$ are predicted by increasing the Forchheimer coefficient $F$, i.e., the inertial force with a larger Forchheimer coefficient forces more gas from the porous layer to the flow duct. Consequently, more heat induced by the retarded flow can be brought into the duct from the heated wall, and the convective heat transfer $\mathrm{Nu}$ decreases, see Fig. $6 \mathrm{~b}$.

The impact of the thickness ratio $h_{r}$ of the porous layer on $f_{\text {app }} \mathrm{Re}$ and $\mathrm{Nu}$ is presented in Figs. $7 \mathrm{a}$ and $\mathrm{b}$ at a fixed Forchheimer coefficient $\left(F=10^{4}\right)$. In this study, the heights of the gas flow duct and solid current collector are kept constant. The thickness ratio $h_{r}$, thickness of porous layer over the total height of duct, is approached by varying the thickness of the porous layer and total height of the duct. It is clear that the Forchheimer coefficient $F$ has no effect on $f_{\text {app }} \mathrm{Re}$ and $\mathrm{Nu}$ for the configuration of $10 \%$ porous layer, i.e., both $\mathrm{BD}$ and $\mathrm{BFD}$ models produce the identical results in terms of $f_{\text {app }} \operatorname{Re}$ and $\mathrm{Nu}$, respectively. By increasing the $h_{r}$ to $20 \%$, the impact of the inertia on gas flow and heat transfer can be identified from Fig. 7, as mentioned earlier. A similar conclusion can be drawn for the configuration of $40 \%$, which is not presented in this figure.

The impact of the thermal conductivity ratio $k_{r}$ (effective thermal conductivity $k_{\text {eff }}$ over fluid conductivity $k_{f}$ ) on $\mathrm{Nu}$ is presented in Fig. $8 \mathrm{a}$ for the base case. As expected, almost the same $f_{\text {app }}$ Re value is predicted for different thermal conductivity ratios ( $k_{r}=1$ vs. 10 in this study) for the $\mathrm{BD}$ and BFD models, respectively. On the other hand, for heat transfer, an increase in the thermal conductivity ratio $k_{r}$ increases $\mathrm{Nu}$ for both BD and BFD models. It could be easily understood that the porous layer with high effective thermal conductivity can improve the heat transfer between the 

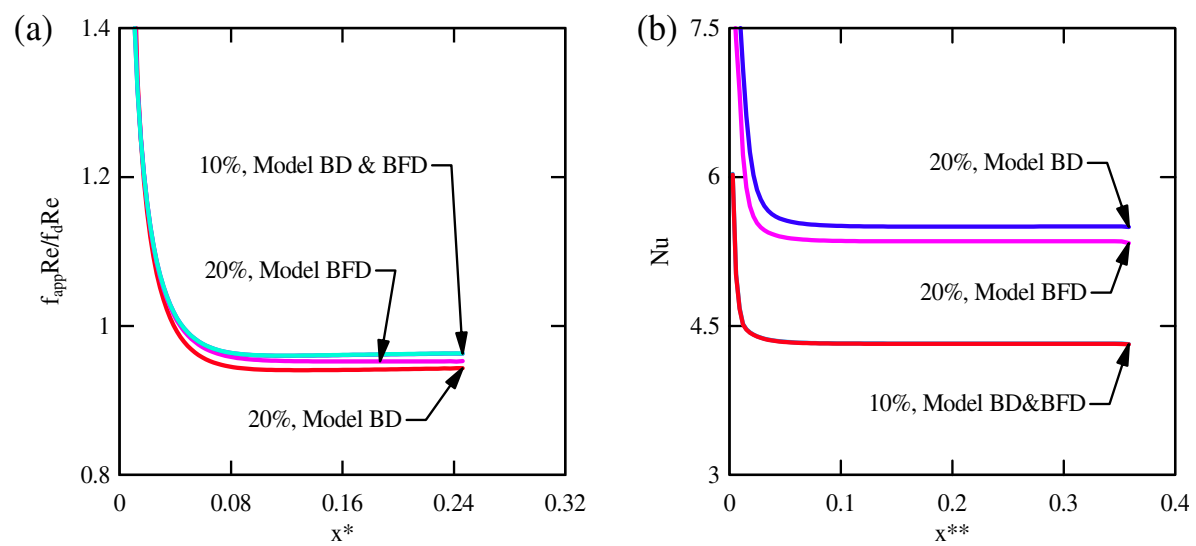

Figure 7. Effects of thickness of porous layer on: (a) $f_{\text {app }} \mathrm{Re}$ and (b) $\mathrm{Nu}$ of cathode duct predicted by BFD models. (View this art in color at www.dekker.com)
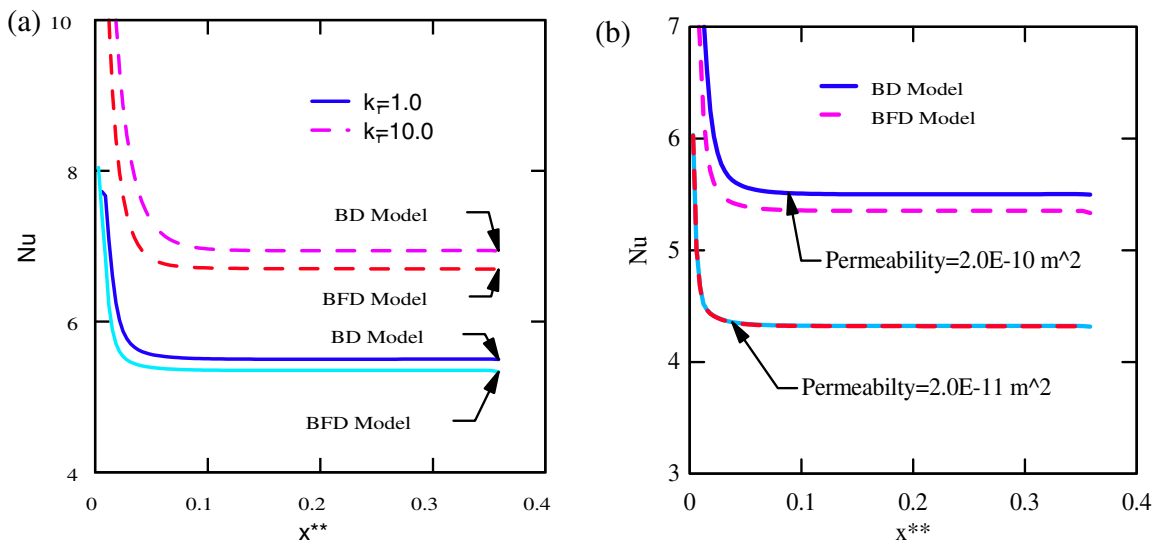

Figure 8. Effects of: (a) thermal conductivity and (b) permeability of porous layer on $\mathrm{Nu}$ for a PEMFC cathode duct (at $F=10^{4}$ ). (View this art in color at www.dekker.com)

heated catalyst surface and fluid by allowing more heat to be conducted. Furthermore, for a fixed $k_{r}$ (both $k_{r}=1$ and 10), the BFD model predicts a smaller $\mathrm{Nu}$ than the BD model does, which is due to the inertial effects as mentioned above. Impacts of porous layer permeability on $\mathrm{Nu}$ are shown in Fig. 8b. It is noticed that by increasing the permeability, $\mathrm{Nu}$ will increase for both BD and BFD models, while $\mathrm{Nu}$ will decrease as the permeability decreases. By comparing with the BD model, the BFD model predicts a smaller $\mathrm{Nu}$ for large permeability $\left(\beta=2.0 \times 10^{-10} \mathrm{~m}^{2}\right)$, as discussed previously. For a small value $\left(\beta=2.0 \times 10^{-11} \mathrm{~m}^{2}\right)$, BD and BFD models predict almost the same results. Obviously, the inertial force has less effect on the heat transfer when the permeability is small. 

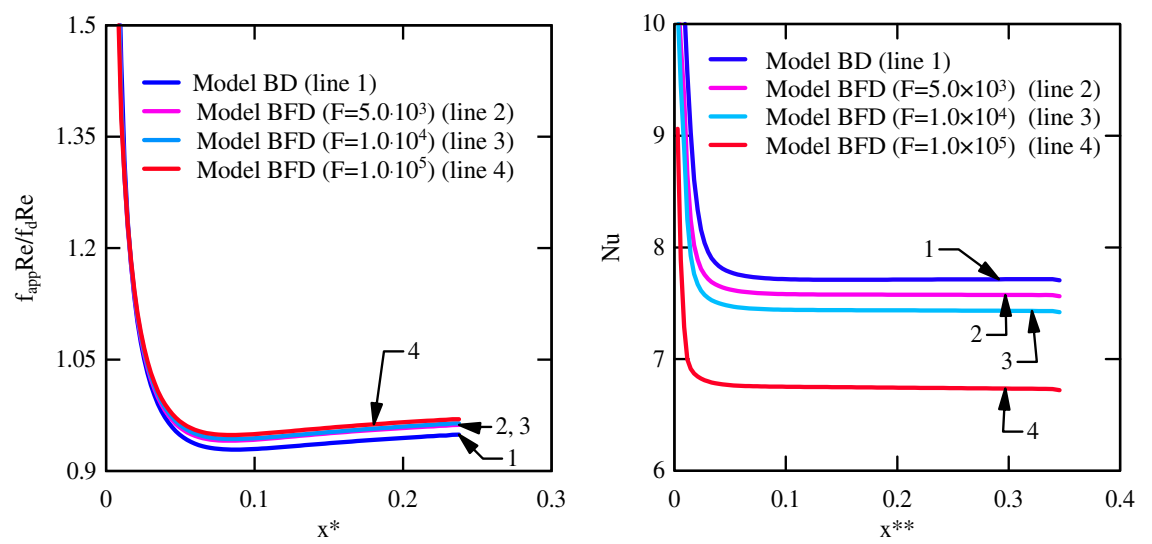

Figure 9. Inertial effects on $f_{\text {app }} \mathrm{Re}$ and $\mathrm{Nu}$ (right) of PEMFC anode duct at base conditions. (View this art in color at www.dekker.com)

As discussed above, the inertial force has effects on the flow field (particularly in the porous layer), and consequently on the heat transfer between the heated catalyst surface and gases in the PEMFC cathode duct. Gas flow and heat transfer in the anode duct have been investigated, and only selected results are presented in Fig. 9 for the inertial effects. As expected, there is a small effect on $f_{\text {app }}$ Re when the inertial coefficient changes, while a significant effect can be found on $\mathrm{Nu}$. By comparing the results from BFD models to those of $\mathrm{BD}$ model, the same conclusions as for the cathode duct can be drawn also, i.e., a large inertial force contributes to more heat injected into the duct, because more gas flow is retarded from the porous media.

In addition, it should be mentioned that parameter studies have been conducted for the thermal conductivity of the solid current collector $k_{s}$, net water transfer coefficient $\alpha$, cell voltage $V_{\text {cell }}$. All the parameters mentioned above give similar results by BD and BFD models. Effects of the interfacial conditions, implemented in this study, between a porous layer and gas flow region have been reported in Yuan et al. (2003). The heat generation caused by the electrochemical reaction and mass transport $\left(\mathrm{H}_{2}\right.$ and $\mathrm{H}_{2} \mathrm{O}$ consumption in anode side, $\mathrm{O}_{2}$ consumption and $\mathrm{H}_{2} \mathrm{O}$ generation in cathode side) have been numerically studied and reported also in Yuan (2003) and Yuan et al. (2003). All these results are not shown or repeated in this study.

\section{CONCLUSIONS}

This study presents simulation and analysis of gas flow and heat transfer, in terms of the apparent friction factor $f_{\text {app }} \mathrm{Re}$ and Nusselt number $\mathrm{Nu}$, in PEMFC anode and cathode ducts. Porous layers appear in the composite ducts under study. The generalized BFD model was employed by including an extra term in the momentum equation to account for the inertial effects. 
Comparisons of the results from the generalized BFD model with those of the generalized BD model are reported. The effects of the porous layer thickness, permeability, inertial coefficient, and ratio of the effective thermal conductivity of the porous medium to the fluid thermal conductivity are also provided. By comparing the $\mathrm{BD}$ and $\mathrm{BFD}$ models, it was found that permeability, inertial coefficient, and thermal conductivity ratio have a significant impact for both cathode and anode ducts for a fixed porous layer configuration. In particular the heat transfer $(\mathrm{Nu})$ between the heated catalyst surface and gases was affected. For this specific PEMFC case, the results show that the inertial effect can be eliminated for small permeability $\left(2 \times 10^{-11}\right)$ or/and for thin porous layer configuration $(10 \%)$.

The current study may be treated as an improved modeling procedure for understanding and prediction of gas flow and heat transfer in PEMFC ducts. The assumptions such as considering only constant porosity (permeability) of the porous medium, and single-phase flow of the water transport are the limitations of this study. However, a first study to include two-phase flow in the cathode duct has been conducted and reported in another article.

\section{NOMENCLATURE}

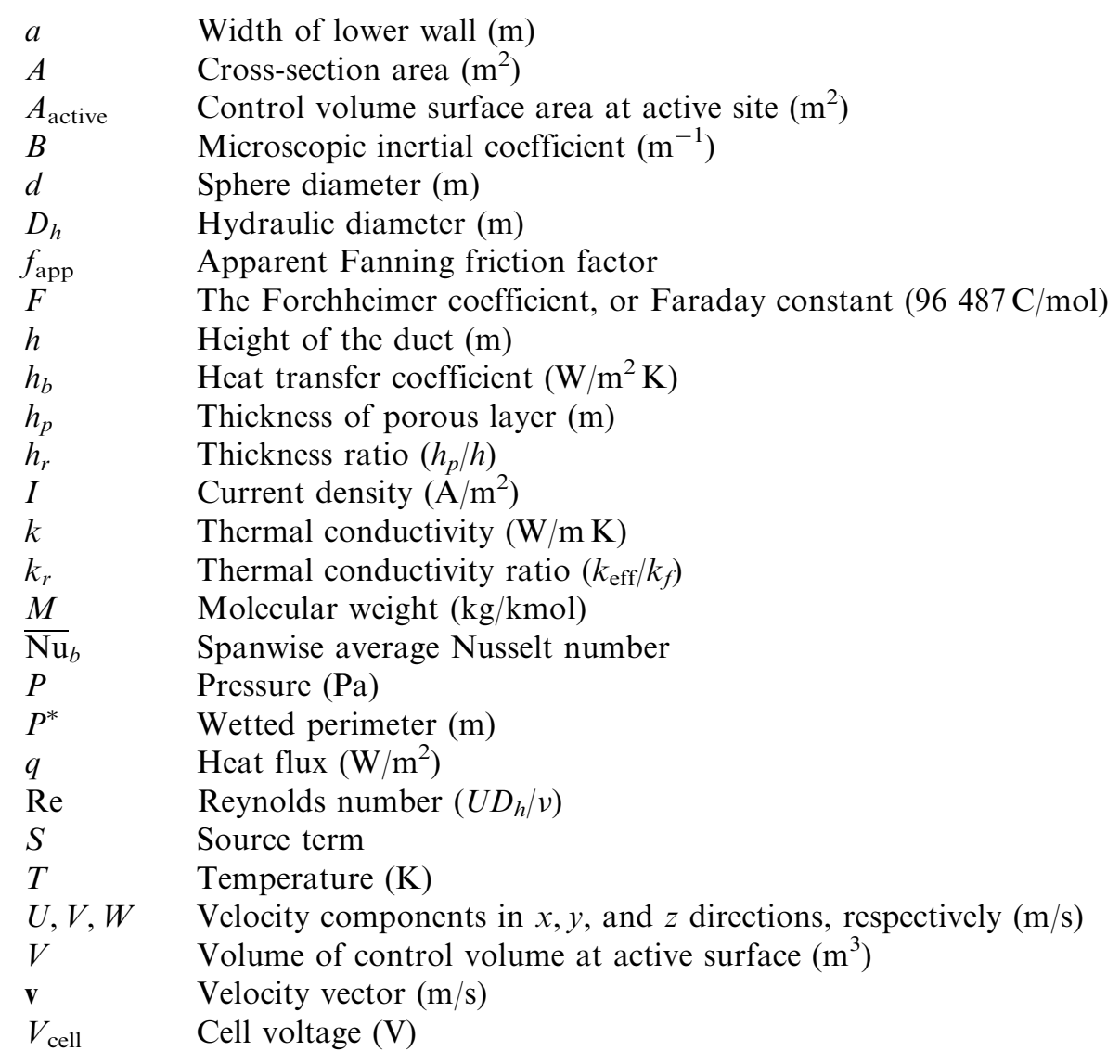


$\begin{array}{ll}V_{m} & \text { Mass transfer velocity at bottom wall }(\mathrm{m} / \mathrm{s}) \\ x, y, z & \text { Cartesian coordinates } \\ x^{*} & \text { Hydrodynamic dimensionless axial distance } \\ x^{* *} & \text { Thermal dimensionless axial distance }\end{array}$

\section{Greek Symbols}

$\alpha \quad$ Net water transport coefficient per proton

$\beta \quad$ Permeability of diffusion layer $\left(\mathrm{m}^{2}\right)$

$\varepsilon \quad$ Porosity

$\mu \quad$ Dynamic viscosity $(\mathrm{kg} / \mathrm{m} \mathrm{s})$

$v \quad$ Kinematic viscosity $\left(\mathrm{m}^{2} / \mathrm{s}\right)$

$\rho \quad$ Density $\left(\mathrm{kg} / \mathrm{m}^{3}\right)$

\section{Subscripts}

$\begin{array}{ll}a & \text { Anode } \\ b & \text { Bottom wall } \\ \text { bulk } & \text { Bulk fluid condition } \\ c & \text { Cathode } \\ \text { di } & \text { Diffusion layer } \\ \text { eff } & \text { Effective parameter } \\ f & \text { Fluid } \\ \mathrm{H}_{2} \mathrm{O} & \text { Water vapor } \\ \text { in } & \text { Inlet } \\ m & \text { Mass transfer } \\ s & \text { Solid current collector }\end{array}$

\section{ACKNOWLEDGMENTS}

The National Fuel Cell Programme by the Swedish Energy Agency financially supported the current research.

\section{REFERENCES}

Alazmi, B., Vafai, K. (2001). Analysis of fluid flow and heat transfer interfacial conditions between a porous medium and a fluid layer. Int. J. Heat Mass Transfer 44:1735-1749.

Alkam, M. K., Al-Nimr, M. A., Hamdan, M. O. (2001). Enhancing heat transfer in parallel-plate channels by using porous inserts. Int. J. Heat Mass Transfer 44:931-938. 
Bejan, A. (1987). Convective heat transfer in porous media. In: Kakac, S., Shah, R. K., Aung, W., eds. Handbook of Single-Phase Convective Heat Transfer. New York: J. Wiley \& Sons.

Chikh, S., Bounedien, A., Bouhadef, K. (1995). Non-Darcian forced convection analysis in an annulus partially filled with a porous material. Num. Heat Transfer 28:707-722.

Comiti, J., Sabiri, N. E., Montillet, A. (2000). Experimental characterization of flow regimes in various porous media-III: limit of darcy's or creeping flow regime for newtonian and purely viscous non-Newtonian fluids. Chem. Eng. Sci. 55:3057-3061.

Dutta, S., Shimpalee, S., Zee, J. W. V. (2000). Three-dimensional numerical simulation of straight channel PEM fuel cells. J. Appl. Electrochemistry 30:135-146.

Lavric, I., Staiti, P., Novak, P., Hocevar, S. (2001). Computational fluid dynamics study of phosphotungstic electrolyte-based fuel cell (PWAFC). J. Power Sources 96:303-320.

Marafie, A., Vafai, K. (2001). Analysis of non-Darcian effects on temperature differentials in porous media. Int. J. Heat Mass Transfer 44:4401-4411.

Poulikakos, D., Renken, K. (1987). Forced convection in a channel filled with porous medium, including the effects of flow inertial, variable porosity, and Brinkman friction. ASME J. Heat Transfer 109:880-888.

Shimpalee, S., Dutta, S. (2000). Numerical prediction of temperature distribution in PEM fuel cells. Num. Heat Transfer (Part A) 38:111-128.

Teng, H., Zhao, T. S. (2000). An extension of Darcy's law to non-Stokes flow in porous media. Chem. Eng. Sci. 55:2727-2735.

Vafai, K., Kim, S. J. (1990). Fluid mechanics of the interface region between a porous medium and a fluid layer-an exact solution. Int. J. Heat and Fluid Flow 11:254-256.

Vafai, K., Kim, S. J. (1994). On the limitations of the Brinkman-Forchheimerextended darcy equation. Int. J. Heat and Fluid Flow 16:11-15.

Yi, J. S., Nguyen, T. V. (1998). An along-the-channel model for proton exchange membrane fuel cells. J. Electrochem. Soc. 145:1149-1159.

Yuan, J. (2003). Computational Analysis of Gas Flow and Heat Transport Phenomena in Ducts Relevant for Fuel Cells. Doctoral thesis, ISBN 91-6285540-9/ISSN 0282-1990, Feb, Lund Institute of Technology Sweden.

Yuan, J., Rokni, M., Sundén, B. (2003). A numerical investigation of gas flow and heat transfer in proton exchange membrane fuel cells. Num. Heat Transfer 44:255-280. 
